$\mathbb{T}$ periodica polytechnica

Electrical Engineering and Computer Science

$57 / 3(2013) 77781$

doi: 10.3311/PPee.2145

http://periodicapolytechnica.org/ee

Creative Commons Attribution (1)

RESEARCH ARTICLE

\section{Compensation of Analog-to-Digital Converter Nonlinearities using Dither}

\author{
Balázs Renczes / István Kollár
}

Received 2013-06-07, revised 2013-06-14, accepted 2013-06-14

\begin{abstract}
Analog-digital converters are inherently nonlinear. Conventional A/D conversion allows no real remedy afterwards. However, an alternative is to increase the available information by observing the transition instants, even with a conventional ADC. Performing the conversion with significant oversampling data points at threshold level crosses can be used. The values of these samples are precisely known, assuming histogram test was executed on the ADC beforehand. For almost constant signals having too few transition level crossings, dither is added. Interpolation is utilized to ensure uniformly sampled data points. This method reduces the conversion error considerably.
\end{abstract}

\section{Keywords}

A/D conversion $\cdot$ nonlinearities $\cdot$ dither $\cdot$ oversampling $\cdot$ linearization · interpolation

\section{Acknowledgement}

The authors would like to thank National Instruments Hungary for providing the ADC hardware.

\section{Balázs Renczes}

Budapest University of Technology and Economics, Magyar tudósok krt. 2., H-1117 Budapest, Hungary

e-mail: Renczes.Balazs@mit.bme.hu

\section{István Kollár}

Budapest University of Technology and Economics, Magyar tudósok krt. 2., H-1117 Budapest, Hungary

e-mail:kollar@mit.bme.hu

\section{Introduction}

Analog-digital converters play a major role in signal processing. Ideally, they would generate samples that unambiguously correspond to the input signal but real converters suffer of distortions caused by ADC nonlinearities (integral and differential nonlinearities), and the finite resolution of the ADC.

The purpose of this paper is to reduce the distortions of analog-to-digital converters, utilizing a novel method. Conventionally, we are looking for amplitude values at the sampling instants. In this case we can only know that the signal was in a given interval (i.e. in a quantization box), but we do not have any more information. This paper approaches the problem in an alternative way: time instants are sought at which threshold level crossings have occurred. Even with a classical ADC, by significant oversampling the threshold crossing times can be determined with relatively high accuracy, and if the threshold levels are known from preceding histogram analysis, from the precisely known transition level - sampling instant pairs the original signal can be restored more precisely than by conventional ways.

\section{Description of the procedure}

The method is based on the idea of so-called asynchronous $A / D$ converters, described in [3]. The basic idea was that the original signal can be restored using some comparators that are set to different comparison levels. At comparison level crossings, the amplitude of the signal is quite precisely known (it equals to the comparison level of the comparator), and these data points can be used to restore the original signal (see Fig. 11).

The method described in this paper is quite similar to asynchronous conversion. The fundamental difference is that instead of comparators a synchronous $\mathrm{A} / \mathrm{D}$ converter with significant oversampling is set in to determine the instants of the threshold level crossings. In this way an existing converter can be improved by significantly reducing its linearity errors.

This way, both time and amplitude values of the samples kept are precisely known, considering the followings. Due to significant oversampling the instants of the level crossings can be captured with high accuracy. On the other hand, the threshold 




Fig. 1. Illustration of asynchronous $A / D$ conversion

levels can also be exactly known, assuming that the converter has been calibrated beforehand. This can be performed using histogram test described in [1]. An implementation of the calibration is given in [2].

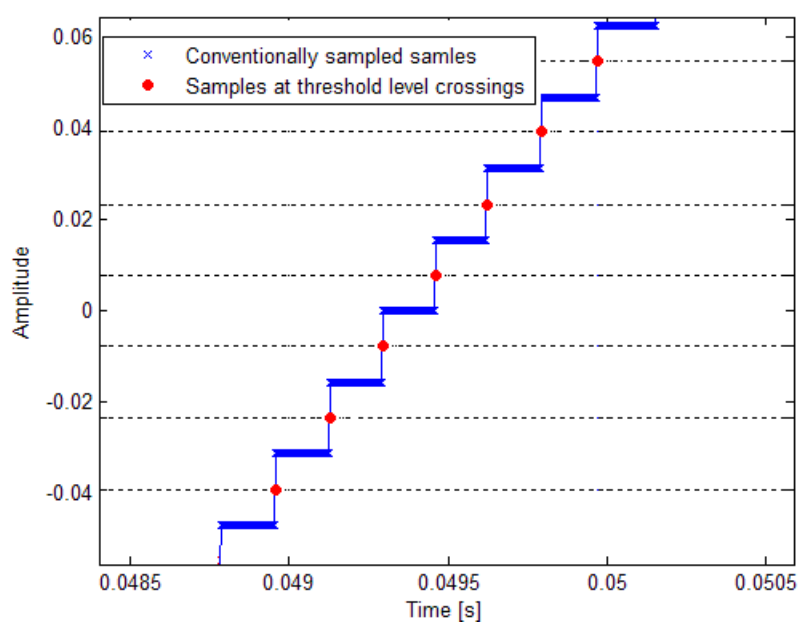

Fig. 2. Illustration of the conversion approach

The method is illustrated in Fig. 2. It is clear that the input signal is significantly oversampled but most of the samples do not contain any additional information about the input, we only know that it was somewhere between two threshold levels. But at code transitions the value of the input signal is quite precisely known. It follows that using these samples the resolution of the A/D can be improved. Furthermore, if calibration has been performed, the integral nonlinearity is also compensated because instead of using the nonlinear digital output value of the converter we can make use of the knowledge of the real threshold values.

These advantages are indisputable, but we certainly have to face several issues due to implementation. First of all, the samples at threshold level crossings are not equally spaced in time, since transitions can occur any time. On the other hand, having low frequency or DC signal, the input may not cross threshold levels enough times so that the input can even theoretically not be restored. The first problem can be solved by interpolation and the second one by adding dither to the input. These are described in Section 3

\section{Solution of issues caused by novel conversion}

\subsection{Non-equally spaced samples}

As mentioned in the previous section, samples at code transitions are not equally spaced in time. Therefore while utilizing uniform sampling ensures that we know the time instant of the sampling (it can be calculated from the ordinal number of the sample), using this novel approach the time instants of the level crossings are also to be saved. Furthermore, digital signal processing algorithms are mostly expecting uniformly sampled data while our method does not provide these. The issue is illustrated in Fig. 3. It has to be mentioned that although the samples after threshold level crossings are not equally spaced in time, they are located on the original equidistant time grid since these samples are a subset of the original samples. For improved accuracy, one can assume that level crossings occurred half way between two neighboring samples with different digital output.

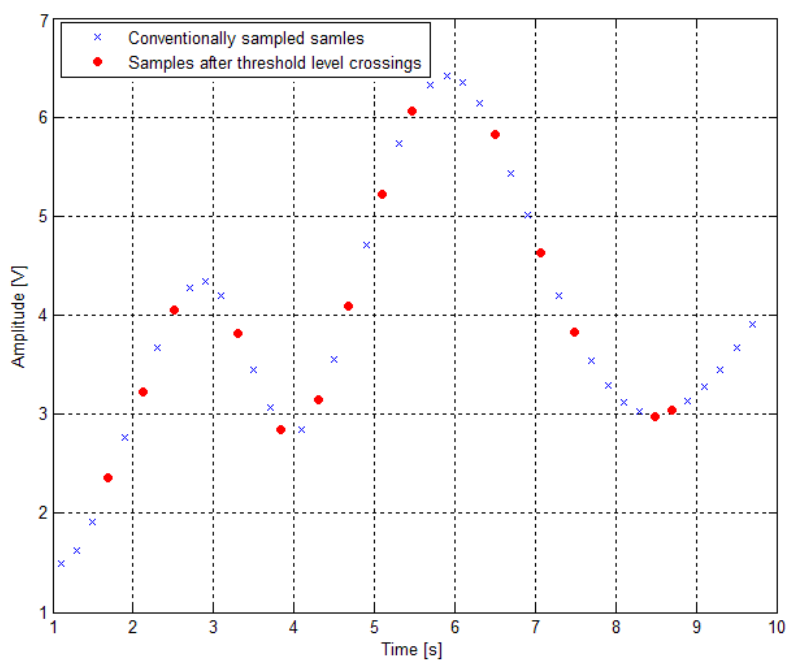

Fig. 3. Illustration of non-equally spaced samples

Interpolating the samples with precisely known values can regenerate data on the original time grid (or to another uniform grid). The simplest interpolation method is linear interpolation but we discarded this because of its distortion. A possible solution for the problem would be utilizing splines. This method fits low-degree polynomial together so that the function's derivative at fitting points is continuous.

Another applicable method which will be applied in this paper is Lagrange interpolation. This method uses $\mathrm{n}$ data points to fit an $(n-1)$ degree polynomial to them. During analog-to-digital conversion a huge number of data points is to be handled. To have limited computational demand, windowing technique can be applied. In this e.g. a 4-point window can be used and the interpolation can be executed for four data points. After that the middle part is kept and the window is shifted forward (see Fig. 4). The interpolated function can be created as the sum of these parts.

Applying this method does not assure that the derivative of the interpolated function is continuous, i.e. the function may have breaks at the joining points but it can still follow the original 
analog signal with relatively high accuracy.



Fig. 4. Illustration of the windowing technique

\subsection{Low-frequency and DC signals}

To launch interpolation we need enough data points. When a low-frequency or DC signal is digitized, often not enough threshold crosses occur and therefore we will not have enough samples. What is more, the number of these data points may not even satisfy the Shannon-theorem, resulting that the original analog signal cannot be restored.

To ensure a sufficient number of threshold level crossings, a small-amplitude dither can be added to the analog signal. Traditionally, dither is utilized to decrease the distortion of quantization - here this is a new use. In [5] detailed information about dithers can be found. In this work the aim is to "push" the input through threshold levels. Regarding the signal to be digitized, the added dither is a noise which increases error at the digital side. To avoid this, dither can be subtracted after digitization. This is the so-called subtractive dither technique. A possible solution for this is that the dither is generated using a D/A converter. If so, we know the value of it quite precisely. Another method is that using a two-channel A/D both the sum of the input signal and the dither and the (amplified) dither are digitized.

Several dither types are known. For the purpose of this work triangular dither seems to be the most appropriate choice since this has constant steepness (at least the absolute value of the steepness is constant). This feature is advantageous because if the steepness were varied, the level crossings would occur more randomly, i.e. for some parts of the input signal there would be many and for other parts only a few samples to interpolate.

\section{Input signals}

As described above, the approach needs oversampling. To ensure this the frequency band of the input has to be limited. Therefore it is assumed that the input signal is bandlimited.

For testing, we should create the worst case situation for the conversion. This is the case at which this approach gives the highest conversion error. This error originates from the fact that despite oversampling the exact time instant of the threshold level crossing cannot be determined and between the level crossing and the perception of it the input signal may change. Assuming that the input is significantly oversampled, the amplitude error can be given as

$$
e_{a} \approx y^{\prime}\left(t_{\text {cross }}\right) e_{t},
$$

where $e_{a}$ and $e_{t}$ are the amplitude and timing errors, respectively, and $y$ is the sum of the input signal and the dither. The worst case situation for this conversion is an input with highest steepness.

Considering all bandlimited signals having the same power, a sine wave with the frequency of the bandlimit has the greatest power density at bandlimit frequency (since all the power of the sine concentrates at this frequency). Derivation in time domain is equivalent with multiplication by $j \omega$ in frequency domain. For other bandlimited signals the power density is "smeared" between 0 and the bandlimit. Since the spectrum is multiplicated by $j \omega$, at frequencies lower than the bandlimit the power density spectrum components are multiplied by lower values than the component at the bandlimit frequency. As a result, a sine with the bandlimit frequency has the maximum-power derivative among bandlimited signals.

Let us briefly consider an example. Maximum (average) steepnesses of bandlimited noise and sine wave with the same power will be compared.

a. Bandlimited noise in frequency band $(-B, B)$ with standard deviation $\sigma$. The variance is $\sigma^{2}$, the power spectral density (PSD) is $\sigma^{2} /(2 B)$ (its integral gives back $\sigma^{2}$ ) (see Fig. 5a).

b. A sine wave of frequency $B$ has the same power as the noise with amplitude $A=\sqrt{2} \sigma$.

We can now calculate the powers of the derivatives (that is, the variances):

$$
\begin{aligned}
P_{\text {whitenoise, derivative }}= & \int_{-\infty}^{\infty}\left|S_{\text {whitenoise, derivative }}(f)\right| d f= \\
= & \int_{-B}^{B} \frac{\sigma^{2}}{2 B}(2 \pi f)^{2} \cdot d f=\frac{4 \pi^{2} B^{2} \sigma^{2}}{3}
\end{aligned}
$$

and

$$
\begin{gathered}
P_{\text {sine,derivative }}=\int_{-\infty}^{\infty}\left|S_{\text {sine, derivative }}(j \omega)\right| d \omega= \\
=\frac{(2 \pi B)^{2} A^{2}}{2}=3 P_{\text {whitenoise, derivative }} .
\end{gathered}
$$

The example shows that the power of the derivative of the maximum frequency sine wave is higher than that of the same-power bandlimited white noise in the same frequency band.

As a result, sine with the bandlimit frequency has $\sqrt{3}$ times the average steepness of the bandlimited white noise. Similar analysis proves that among same-power bandlimited signals, the maximum-frequency sine wave has the maximum-power derivative.

On the other hand, a sine wave seems to be an appropriate choice for a test signal, since after conversion the error of the digitized signal wave can easily be determined e.g. by using LS sine wave fit. 
Fig. 5. Power density spectrums of bandlimited

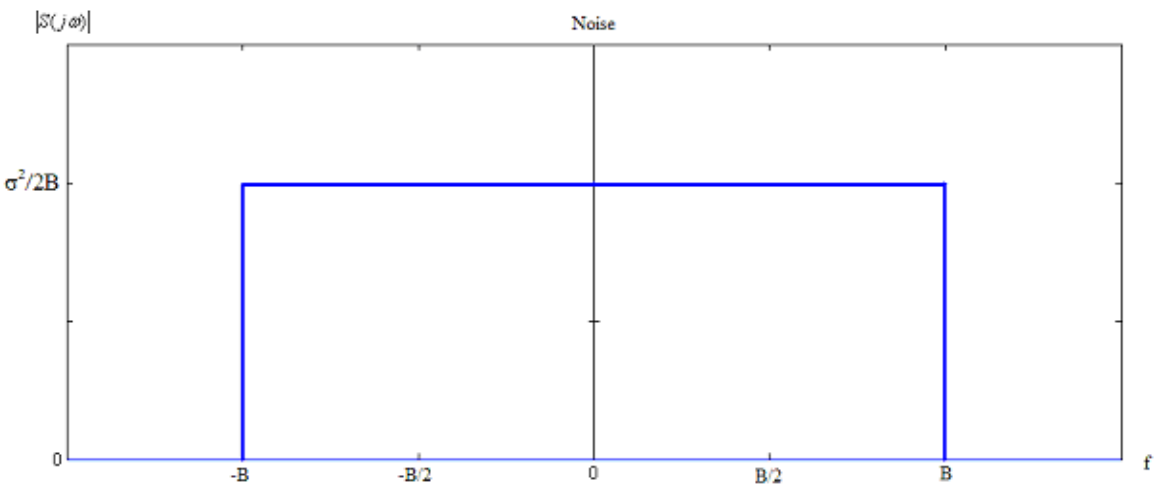

a)

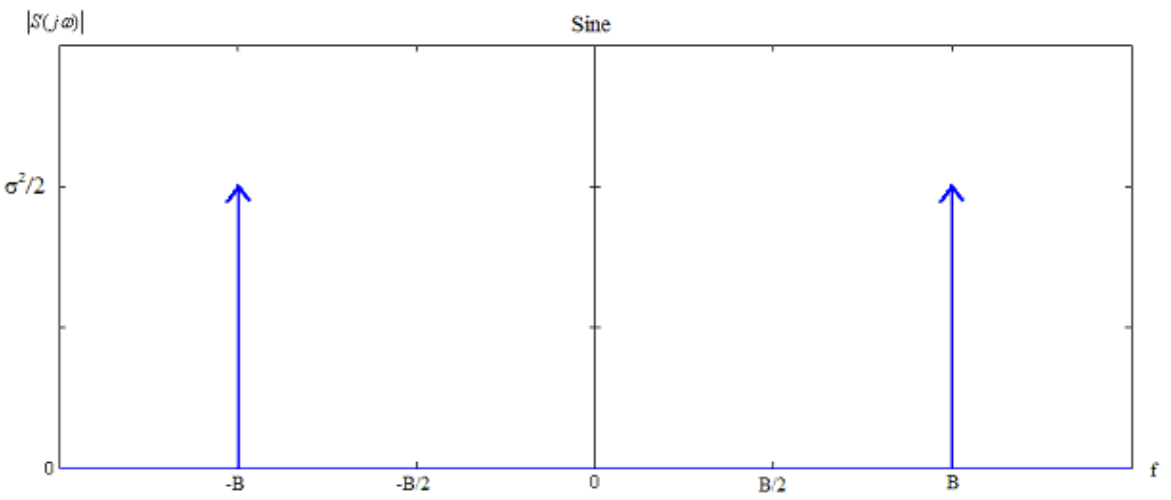

b)

\section{Parameter settings for measurement}

For the measurement the analog-digital converter myDAQ of National Instruments (resolution 16 bit, input range $\pm 2 \mathrm{~V}$, maximum sampling frequency $200 \mathrm{kHz}$ ) was used.

The RMSE (root mean square error) of digitizing a sinusoidal input without dither addition can be calculated, considering the followings. The maximum derivative value is $2 \pi f_{x} A_{x}$, where $x$ refers to the sine wave. Since the derivative function of a sinusoidal function is cosinusoidal, the effective value of the derivative is $\sqrt{2} \pi f_{x} A_{x}$. We know that the code transition occurred between two sampling instants. Assuming that the timing error is uniformly distributed between $-T_{C L K} / 2$ and $T_{C L K} / 2$ it can be calculated as $\frac{T_{C L K}}{2 \sqrt{3}}$. Using Eq. (1) we can now calculate the RMSE value which is

$$
\frac{1}{\sqrt{6}} \pi f_{x} A_{x} T_{C L K} \approx 0.41 \pi f_{x} A_{x} T_{C L K}
$$

We can also determine how many threshold level crossings occur during a sampling period. Or, inversely, a code transition occurs after how many sampling periods on average. Keeping the former value low is of importance because when the input signal changes too fast then restoring the signal with high accuracy is not possible. The maximum number of level crossings is given by Eq. 5, [6]

$$
n_{\text {max }}=\frac{\Delta y_{\text {max }}}{L S B}=\frac{2 \pi A_{x} \frac{f_{x}}{f_{C L K}}+4 A_{d} \frac{f_{d}}{f_{C L K}}}{L S B}
$$

where $d$ refers to the dither. To keep this value low only the 8 most significant bits of the 16 bit ADC were used. The given $n_{\max }$ is roughly independent of the input signal, if the slew rate of the dither is much higher than that of the sine.

The sampling frequency may be set to be maximum (200 $\mathrm{kHz}$ ). If the input signal is sinusoidal, then it has a sufficient number of threshold level crossings, so no dither has to be added to execute the method. With frequency of $500 \mathrm{~Hz}$ and amplitude of $0.5 \mathrm{~V}$ the maximum threshold level crossings can be computed using Eq. (5) : $n_{\max }=0.5027$. This means that after minimum 1.99 sampling periods a transition occurs. Using these settings the RMSE value can also be determined using Eq.(4) :

$$
R M S E_{\text {theoretical }}=\frac{L S B}{9.75}
$$

The PQN-model (Pseudo Quantization Noise Model) states that the quantization noise can be considered as white noise, which is uniformly distributed between $-L S B / 2$ and $L S B / 2$. The PQN-model is described in details in [5]. In the model RMSE is $\frac{L S B}{2 \sqrt{3}}$. Using the novel approach and the given parameter settings RMSE can be reduced to about the third of it (see Eq.(6).).

\section{Verification by measurement}

With the given settings a measurement can be carried out. If dither is also added to ensure adequate number of level crossings, two channels of the ADC is to be used. The first channel samples the sum of the dither and the sine wave, while the second one samples the dither itself. To ensure that summing of the two signals does not affect the measured value of the dither, an operational amplifier is added to the circuit. Measurement 
Fig. 6. Measurement settings

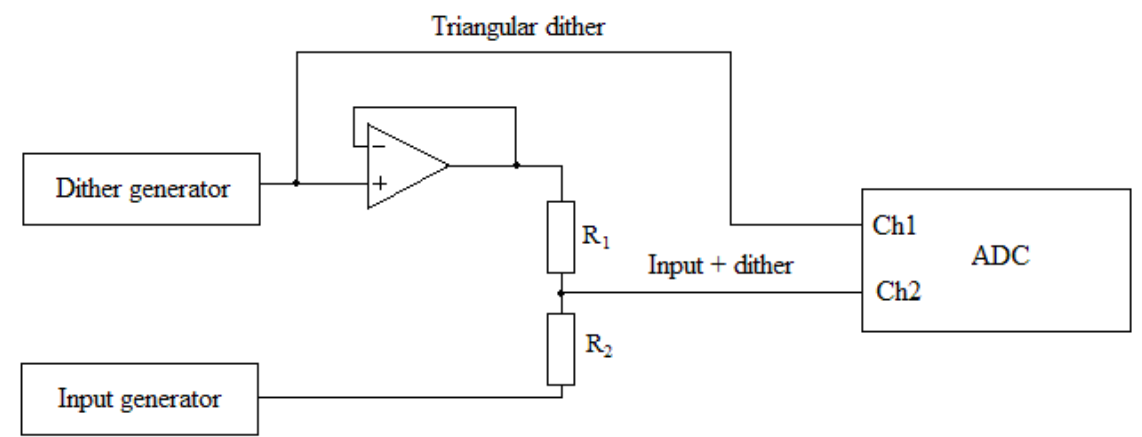

settings can be seen in Fig. 6. Properly choosing the value of resistors R1 and R2 allows measuring the dither with higher precision in channel one, while in the sum of the two signals only the divided part of the original triangular signal appears. As mentioned in Section 5, the sine wave has sufficient slope, thus in this measurement the use of the dither was not necessary.

The method is illustrated in Fig. 7. In this the threshold levels were counted as the average of the two codes before and after transition. The figure shows that the interpolated signal follows the sinusoidal form much better than the usual staircase function.

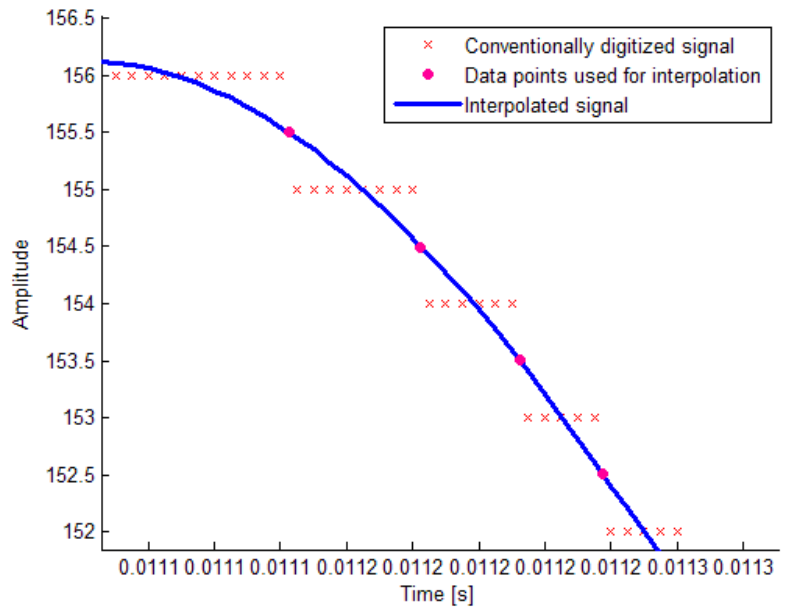

Fig. 7. Comparison of samples of a sine wave measured with traditional ADC and reconstructed samples with the proposed method

To compensate the nonlinearities of the ADC, the method was also executed with threshold levels given by the histogram test. After the measurement LS sine wave fit was carried out to check the quality of the conversion. The RMSE of the signal's fit equals to $\frac{L S B}{11.64}$ which is even lower than the theoretical value, but it is to be mentioned that error given in Eq. (6) is calculated only for data points that were interpolated and the interpolation itself reduces the error on average. RMSE can also be given only for the interpolated data points and it equals to $\frac{L S B}{9.30}$ which is now close to the theoretical value.

\section{Conclusions}

It has been shown that by significant oversampling and interpolation, the nonlinearities of an A/D converter like finite res- olution and integral nonlinearity can be considerably reduced. Unlike conventional conversion during this novel approach only data points are kept that were recorded at threshold level crossings. The advantage of the method is that for these data points the amplitudes can be determined precisely if the calibration of the converter had been performed beforehand. In this way, the analog signal can be restored with higher accuracy than by conventional ways. The method compensates for the integral nonlinearity of the converter. Furthermore, the resolution of the A/D is enhanced, as well.

\section{References}

1 Blair $\mathbf{J}$, Histogram measurement of ADC nonlinearities using sine waves, IEEE Transactions on Instrumentation and Measurement, 43(3), (Jun. 1994), 373-383.

2 Virosztek T, Kollár I, User-Friendly Matlab Tool for Easy ADC Testing, 19th IMEKO TC 4 Symposium and 17th IWADC Workshop: Advances in Instrumentation and Sensors Interoperability, (2013). Paper 133.

3 Wang T, Wang D, Hurst PJ, Levy BC, Lewis SH, A Level-Crossing Analog-to-Digital Converter with Triangular Dither, IEEE Transactions On Circuits and Systems, 56(9), (Sep. 2009), 2089-2099.

4 Sayiner N, Sorensen HV, Viswanathan TR, A New Signal Acquisition Technique, Proc. 35th Midwest Symp. Circuits Syst., 2, ( Aug. 1992), 11401142.

5 Widrow B, Kollár I, Quantization Noise: Roundoff Error in Digital Computation, Signal Processing, Control, and Communications, Cambridge University Press; Cambridge, UK, 2008.

6 Renczes B, Kollár I, Linearization of $A / D$ converters using interpolation of samples, 19th IMEKO TC 4 Symposium and 17th IWADC Workshop: Advances in Instrumentation and Sensors Interoperability, (2013). Paper 124. 